\title{
Functional and cognitive outcomes after COVID-19 delirium
}

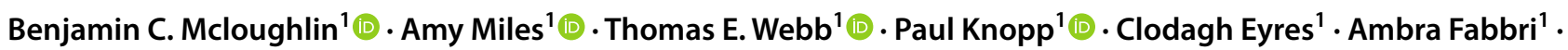 \\ Fiona Humphries ${ }^{1} \cdot$ Daniel Davis $^{1,2}$ (1)
}

Received: 27 May 2020 / Accepted: 2 July 2020 / Published online: 14 July 2020

(c) The Author(s) 2020

\section{Key summary points}

Aim To investigate functional and cognitive outcomes among patients with delirium in COVID-19.

Findings Delirium in COVID-19 was prevalent (42\%), but only a minority had been recognised by the clinical team. At 4-week follow-up, delirium was significantly associated with worse functional outcomes, independent of pre-morbid frailty. Cognitive outcomes were not appreciably worse.

Message The presence of delirium is a significant factor in predicting worse functional outcomes in patients with COVID-19.

\begin{abstract}
Purpose To ascertain delirium prevalence and outcomes in COVID-19.

Methods We conducted a point-prevalence study in a cohort of COVID-19 inpatients at University College Hospital. Delirium was defined by DSM-IV criteria. The primary outcome was all-cause mortality at 4 weeks; secondary outcomes were physical and cognitive function.

Results In 71 patients (mean age 61, 75\% men), 31 (42\%) had delirium, of which only 12 (39\%) had been recognised by the clinical team. At 4 weeks, 20 (28\%) had died, 26 (36\%) were interviewed by telephone and 21 (30\%) remained as inpatients. Physical function was substantially worse in people after delirium -50 out of 166 points $(95 \% \mathrm{CI}-83$ to $-17, p=0.01$ ). Mean cognitive scores at follow-up were similar and delirium was not associated with mortality in this sample.

Conclusions Our findings indicate that delirium is common, yet under-recognised. Delirium is associated with functional impairments in the medium term.
\end{abstract}

Keywords COVID-19 $\cdot$ Delirium $\cdot$ Telephone interview for cognitive status $\cdot$ Barthel Index $\cdot$ Nottingham extended activities of daily living

\section{Introduction}

Delirium is one of the most common acute disorders in general hospitals, affecting around $25 \%$ of older patients [1]. Delirium is closely linked with adverse outcomes, including

Electronic supplementary material The online version of this article (https://doi.org/10.1007/s41999-020-00353-8) contains supplementary material, which is available to authorized users.

Daniel Davis

daniel.davis@ucl.ac.uk

1 Department of Medicine for the Elderly, University College London Hospitals NHS Foundation Trust, London, UK

2 Department of Population Science and Experimental Medicine, MRC Unit for Lifelong Health and Ageing at UCL, 1-19 Torrington Place, London WC1E 7HB, UK higher mortality, increased length of stay, long-term cognitive and functional decline, and risk of institutionalisation [2,3]. Many screening instruments are available and the 4AT is the one best established within the UK National Health Service [4, 5]. Missed diagnoses may contribute to the excess mortality observed [6,7], making systematic detection of delirium essential in any setting, no less so in the context of COVID-19 infection.

To date, there has been limited work investigating the prevalence of and outcomes relating to delirium in COVID19. Early studies describing the broad neurological features of COVID-19 suggest that $20-30 \%$ of hospitalised patients will present with or develop delirium or mental status changes, increasing to $60-70 \%$ in severe cases [8-10]. We set out to describe the point prevalence of delirium in patients hospitalised with COVID-19, and quantify its 
association with mortality and cognitive and physical impairments at 4 weeks.

\section{Methods}

\section{Study design and setting}

We conducted a point prevalence study at University College Hospital of every inpatient (including critical care) with a diagnosis of COVID-19. All assessments for delirium took place on a single day, with outcomes measured 4 weeks later.

\section{Participants}

We included all adult inpatients on Tuesday 21st April 2020 who had tested positive for SARS-CoV-2 on combined throat and high nasal swab reverse-transcriptase polymerase chain reaction (RT-PCR). We did not include participants with a clinical suspicion of COVID-19 (e.g. on radiological or laboratory parameters) who were RT-PCR negative. We excluded patients who were discharged or died prior to the point of assessment.

\section{Outcomes}

The primary outcome was all-cause mortality at 4 weeks. Deaths occurring outside of hospital were captured from the NHS Spine, a centralised national registry. Secondary outcomes were cognitive function and performance in activities of daily living at 4-week telephone follow-up. Cognitive function was measured using the modified Telephone Instrument for Cognitive Status (TICS-m), and performance in activities of daily living measured using a composite of the Barthel Index and Nottingham Extended Activities of Daily Living (NEADL) scores [11-13]

\section{Delirium assessments}

All assessments were carried out on a single day by one rater (BM) with data reviewed by a delirium expert (DD). Delirium was defined by DSM-IV criteria. The 4AT was part of the assessment, with information supplemented by informant history (usually the clinical team) and review of medical notes from the previous $24 \mathrm{~h}$. Therefore, disturbance of consciousness was defined by altered arousal through use of the modified Richmond Agitation-Sedation Scale (mRASS) and/or inattention on 'months of the year backwards' or equivalent task; change in cognition and/ or perceptual disturbance was identified through testing orientation using the AMT4 and components of the mental state examination; fluctuating course and physiological basis was determined by chart review. Where participants were unable to speak English $(n=2)$, we made the diagnosis with the assistance of formal or family interpreters. All delirium cases were classified as hypoactive (reduced alertness), hyperactive (increased alertness or motor agitation), mixed (some features of both hypoactive and hyperactive), or no clear motor subtype.

\section{Other variables}

We recorded additional clinical data: age, sex, ethnicity, dementia status (definite dementia $=$ documented history; probable dementia $=$ no documented diagnosis but history of progressive cognitive impairment affecting activities of daily living; no dementia). Clinical Frailty Scale (CFS) from 1 to 9 was determined by chart review by the geriatric medicine team. We recorded if patients had already been screened for delirium using any recognised tool and if a diagnosis of delirium had been recorded in the medical notes by the usual care team.

\section{Statistical methods}

Differences between patients with and without delirium were analysed using $\chi^{2}$ tests for categorical data and independent $t$ tests for continuous data. We defined cases of delirium as those only meeting all DSM-IV criteria; all other participants were considered to be 'non-delirium' unless they were completely unassessable, because they were highly sedated in critical care. We compared 4-week survival in delirium versus non-delirium using logistic regression (primary outcome). For secondary outcomes, we treated TICS-m and Barthel + NEADL scores as continuous and compared these in people with and without delirium using linear regression, adjusted by age, sex and Clinical Frailty Scale score (as a continuous measure). Post-estimation procedures included examination of all residuals for heteroskedasticity. All analyses were conducted in Stata 14.0 (StataCorp, Texas).

These analyses were conducted as part of a service evaluation project and individual consent was not necessary as determined by the NHS Health Research Authority (HRA), the regulatory body for medical research for England, UK. The HRA has the Research Ethics Service as one of its core functions and they determined the project was exempt from the need to obtain approval from an NHS Research Ethics Committee. https://www.hra.nhs.uk/about-us/committees -and-services/res-and-recs.

\section{Results}

A total of 82 patients were identified, though some were discharged prior to assessment $(n=6)$, had died prior to assessment $(n=3)$, or not present at review $(n=2)$. The final 
sample included 71 patients. Among these, 25 patients were on acute medical wards, 5 in a High Dependency Unit, and 41 in critical care.

The mean age was 61 years (range 24-91), 51 (72\%) were men, $6(9 \%)$ had dementia or probable dementia and median clinical frailty score was 2 (IQR 2,3) (Table 1). Delirium was identified in 31 patients (42\%); hypoactive delirium accounted for $37 \%$ of presentations and $53 \%$ were hyperactive. Only a minority of cases $(n=12,39 \%)$ had been routinely recognised by the treating clinical team. Between delirium and non-delirium patients, 4AT sub-scores were different for each item (Supplementary Table). Where arousal was sufficient to assess directly, patients were evaluated for the presence of added symptoms. Phenomenologically, there were no differences in proportions with hallucinations, delusions, sleep disturbance or distress (Table 1).

At follow-up, 20 (28\%) had died, 21 (30\%) were still inpatients, $26(36 \%)$ were interviewed by telephone, and $4(6 \%)$ could not be contacted (Fig. 1). Of the remaining inpatients, seven remained delirious and eight were still unassessable.

Mean cognitive scores at follow-up were similar in individuals with and without delirium (34.5 and 41.5, out of 53 respectively, $p=0.06$ ) (Fig. 2). However, physical function was substantially worse in people after delirium (97 versus $153, p<0.01)$. These differences were still evident after adjustment for age, sex, HDU/ICU admission status and pre-morbid frailty; here, delirium accounted for -50 out of 166 points ( $95 \% \mathrm{CI}-83$ to $-17, p=0.01$ ) (Table 2 ).

Delirium was not associated with mortality in an age-sex-frailty-adjusted model (OR 6.0, 95\% CI 0.6-60, $p=0.13)$.

\section{Discussion}

In patients hospitalised with COVID-19, delirium was found to be prevalent — but often undetected — and was associated with poor functional outcomes. We did not find many atypical features in this sample, though more hyperactive presentations were apparent than in other case series. There was no evidence of excess mortality or worse cognition at 4 weeks. Taken together, our findings suggest that delirium is a significant clinical complication of COVID-19 and long-term sequelae merits dedicated follow-up.

Our results should be treated with caution. Data were collected at a single site in an urban university hospital and at a single time point, capturing a spectrum of stages in the disease course. As a sample of hospitalised patients, our findings may not be generalisable to community populations. With a substantial number of patients in critical care, there are additional complexities to the ascertainment of delirium. Our measure of physical function was established through self/informant report and direct assessments would have been more accurate. Nonetheless, our data are strengthened by the consistent and systematic approach to delirium detection and robust methods for follow-up.

These findings add to the growing body of work reporting the prevalence of and adverse outcomes associated with delirium (or 'confusion') in COVID-19 [8-10]. Delirium appears to be twice as common in COVID-19 than in other estimates (though these have often excluded patients in critical care) [14]. While adverse cognitive and functional outcomes from delirium are well established, this is the first report to quantify these in the context of COVID-19.
Table 1 Patient characteristics of study participants and delirium status

\begin{tabular}{lllll}
\hline & No delirium $(n=16)$ & Delirium $(n=31)$ & Unassessable $(n=24)$ & $P$ \\
\hline Age (SD) & $63.3(15.1)$ & $65.9(15.3)$ & $55.5(11.8)$ & 0.58 \\
Sex (\%) M & $10(19.6)$ & $25(49)$ & $16(31.4)$ & 0.33 \\
CFS (\%) & & & 0.1 \\
1 & $3(18.8)$ & $5(16.1)$ & $9(37.5)$ & \\
2 & $9(56.3)$ & $11(35.5)$ & $12(50)$ & \\
3 & $0(0)$ & $5(16.1)$ & $2(8.3)$ & \\
4 & $1(6.3)$ & $0(0)$ & $0(0)$ & \\
5 & $1(6.3)$ & $1(3.2)$ & $0(4.2)$ & 0.1 \\
6 & $1(6.3)$ & $7(22.6)$ & $0(0)$ & 0.07 \\
7 & $1(6.3)$ & $2(6.5)$ & 2 & 0.2 \\
Dementia (\%) & $1(16.7)$ & $5(83.3)$ & & 0.68 \\
Hallucinations (\%) & $2(50)$ & $2(50)$ & & 0.33 \\
Delusions (\%) & $0(0)$ & $1(100)$ & & \\
Sleep disturbance (\%) & $10(62.5)$ & $6(37.5)$ & $2(66.7)$ & \\
Distress (\%) & $1(33.3)$ & & & \\
\hline
\end{tabular}

$S D$ standard deviation, Unassessable refers to patients with low mRASS, N/A refers to patients where mRASS was not required i.e. ward patients 
Fig. 1 Flowchart describing patient recruitment, assessment and follow-up
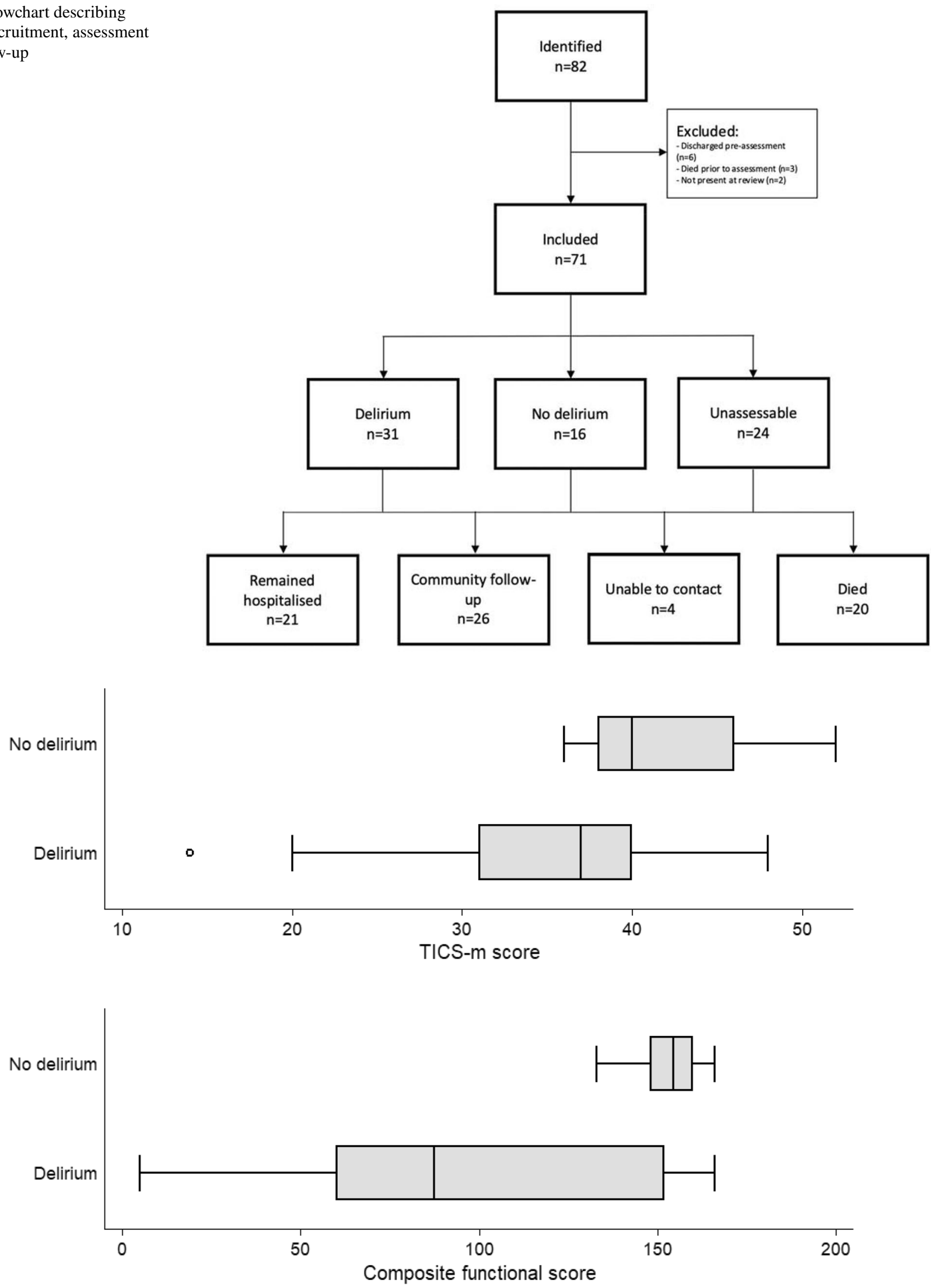

Fig. 2 Cognitive and functional outcomes 4 weeks after delirium ascertainment. TICS-m: modified Telephone Interview for Cognitive Status; Composite functional score formed from Barthel plus Nottingham Extended Activities of Daily Living scale 
Table 2 Univariable and multivariable models estimating the associations with physical function at 4 weeks on a combined Barthel (100 points) and Nottingham Extended Activities of Daily Living score (66 points)

\begin{tabular}{|c|c|c|c|c|c|c|c|c|}
\hline \multirow{3}{*}{ Age } & \multicolumn{4}{|c|}{ Univariable models } & \multicolumn{4}{|c|}{ Multivariable model } \\
\hline & \multirow{2}{*}{$\frac{\mathrm{HR}}{-1.3}$} & \multicolumn{2}{|l|}{$95 \% \mathrm{CI}$} & $P$ & \multirow{2}{*}{$\frac{\mathrm{HR}}{-0.6}$} & \multicolumn{2}{|l|}{$95 \% \mathrm{CI}$} & \multirow{2}{*}{$\frac{P}{0.22}$} \\
\hline & & -2.6 & 0.08 & 0.06 & & -1.7 & 0.4 & \\
\hline Sex & -40.2 & -92 & 11.5 & 0.12 & -12.6 & -47.7 & 22.6 & 0.46 \\
\hline Delirium & -56.5 & -92.3 & -20.8 & 0.004 & -49.7 & -82.9 & -16.5 & 0.01 \\
\hline HDU/ICU & 4.1 & -39.7 & 47.9 & 0.85 & 19.7 & -11.6 & 51.1 & 0.20 \\
\hline CFS & -19 & -27.9 & -10.2 & $<0.01$ & -10.2 & -20.3 & -0.1 & 0.05 \\
\hline
\end{tabular}

$H R$ hazard ratio, $C I$ confidence interval, $H D U / I C U$ patients in high dependency or intensive care unit at baseline delirium assessment, $C F S$ Clinical Frailty Scale
Though its impact seems to be clearer in terms of functional impairment, it is possible that persistent differences in cognitive outcomes would become more apparent with longer follow-up.

The pathophysiology of COVID-19 delirium, and its long-term outcomes, is likely to be multifactorial. Indirect mechanisms such as pyrexia, hypoxia, dehydration, metabolic derangements, and medications may be relevant. Direct pathways could also play a role, in the form of neuroinflammation and vascular injury [15]. These myriad risk factors underscore the importance of comprehensive assessment and management of delirium and its brain complications.

Our findings emphasise the requirement for dedicated delirium detection and management in COVID-19. Clearly, further work is needed to understand the mechanisms leading to delirium and its clinical and epidemiological outcomes. Though it is not known if any adverse sequelae could be mitigated through better delirium care, the scale and potential for distress itself justifies it as a clinical priority.

Acknowledgements Thanks to Helen Bowden, MRC Unit for Lifelong Health and Ageing at UCL, who provided additional training.

Author contributions $\mathrm{BM}, \mathrm{AM}, \mathrm{TW}, \mathrm{PK}, \mathrm{FH}, \mathrm{AF}$, and $\mathrm{CE}$ collected the primary data. DD undertook the statistical analyses and had oversight of the project. BM drafted the first version of the manuscript. All authors contributed to revision and intellectual content of the final submission.

Funding Daniel Davis is funded through a Wellcome Intermediate Clinical Fellowship (WT107467).

Data availability On request.

Code availability On request.

\section{Compliance with ethical standards}

Conflict of interest The authors declare that they have no conflict of interest.

Ethical approval The HRA has the Research Ethics Service as one of its core functions and they determined the project was exempt from the need to obtain approval from an NHS Research Ethics Committee. https://www.hra.nhs.uk/about-us/committees-and-services/res-andrecs/.

Informed consent These analyses were conducted as part of a service evaluation project and individual consent was not necessary as determined by the NHS Health Research Authority (HRA), the regulatory body for medical research for England, UK.

Open Access This article is licensed under a Creative Commons Attribution 4.0 International License, which permits use, sharing, adaptation, distribution and reproduction in any medium or format, as long as you give appropriate credit to the original author(s) and the source, provide a link to the Creative Commons licence, and indicate if changes were made. The images or other third party material in this article are included in the article's Creative Commons licence, unless indicated otherwise in a credit line to the material. If material is not included in the article's Creative Commons licence and your intended use is not permitted by statutory regulation or exceeds the permitted use, you will need to obtain permission directly from the copyright holder. To view a copy of this licence, visit http://creativecommons.org/licenses/by/4.0/.

\section{References}

1. Gibb K, Seeley A, Quinn T, Siddiqi N, Shenkin S, Rockwood $\mathrm{K}$ et al (2020) The consistent burden in published estimates of delirium occurrence in medical inpatients over four decades: a systematic review and meta-analysis study. Age Ageing 49(3):352-360

2. Davis DHJ, Muniz-Terrera G, Keage HAD, Stephan BCM, Fleming J, Ince PG et al (2017) Association of delirium with cognitive decline in late life: a neuropathologic study of 3 population-based cohort studies. JAMA Psychiatry 74(3):244-251

3. Reston JT, Schoelles KM (2013) In-facility delirium prevention programs as a patient safety strategy: a systematic review. Ann Intern Med 158(5 Pt 2):375-380

4. Validation of the 4AT, a new instrument for rapid delirium screening: a study in 234 hospitalised older people https://academic.oup. com/ageing/article/43/4/496/2812210

5. Shenkin SD, Fox C, Godfrey M, Siddiqi N, Goodacre S, Young J et al (2019) Delirium detection in older acute medical inpatients: a multicentre prospective comparative diagnostic test accuracy study of the 4AT and the confusion assessment method. BMC Med 17(1):138

6. Kakuma R, Du Fort GG, Arsenault L, Perrault A, Platt RW, Monette $\mathrm{J}$ et al (2003) Delirium in older emergency department patients discharged home: effect on survival. J Am Geriatr Soc 51(4):443-450 
7. Bellelli G, Nobili A, Annoni G, Morandi A, Djade CD, Meagher DJ et al (2015) Under-detection of delirium and impact of neurocognitive deficits on in-hospital mortality among acute geriatric and medical wards. Eur J Intern Med 26(9):696-704

8. Mao L, Jin H, Wang M, Hu Y, Chen S, He Q, et al (2020) Neurologic manifestations of hospitalized patients with coronavirus disease 2019 in Wuhan, China. JAMA Neurol. https://jamanetwor k.com/journals/jamaneurology/fullarticle/2764549

9. Benussi A, Pilotto A, Premi E, Libri I, Giunta M, Agosti C, et al (2020) Clinical characteristics and outcomes of inpatients with neurological disease and COVID-19. Neurology. https://doi. org/10.1101/2020.04.28.20082735

10. Docherty AB, Harrison EM, Green CA, Hardwick HE, Pius R, Norman L, et al (2020) Features of 20133 UK patients in hospital with covid-19 using the ISARIC WHO Clinical Characterisation Protocol: prospective observational cohort study. BMJ 369. https ://www.bmj.com/content/369/bmj.m1985

11. Buckwalter JG, Crooks VC, Petitti DB (2002) A preliminary psychometric analysis of a computer-assisted administration of the telephone interview of cognitive status-modified. J Clin Exp Neuropsychol 24(2):168-175
12. Shah S, Vanclay F, Cooper B (1989) Improving the sensitivity of the Barthel Index for stroke rehabilitation. J Clin Epidemiol https ://pubmed.ncbi.nlm.nih.gov/2760661/

13. Nouri F, Lincoln N (1987) An extended activities of daily living scale for stroke patients. Clin Rehabil 1(4):301-305

14. Welch C, McCluskey L, Wilson D, Chapman GE, Jackson TA, Treml J et al (2019) Delirium is prevalent in older hospital inpatients and associated with adverse outcomes: results of a prospective multi-centre study on World Delirium Awareness Day. BMC Med 17(1):229

15. Steardo L, Steardo L, Zorec R, Verkhratsky A. Neuroinfection may contribute to pathophysiology and clinical manifestations of COVID-19. Acta Physiologica n/a(n/a):e13473.

Publisher's Note Springer Nature remains neutral with regard to jurisdictional claims in published maps and institutional affiliations. 